\title{
Topographic organization of the cerebral cortex and brain cartography
}

\author{
Simon B. Eickhoff ${ }^{1,2,3}$, R. Todd Constable ${ }^{4,5,6}$, B.T. Thomas Yeo $\mathrm{Y}^{7,8,9}$ \\ ${ }^{1}$ Institute of Systems Neuroscience, Medical Faculty; Heinrich-Heine University Düsseldorf \\ 2 Institute of Clinical Neuroscience and Medical Psychology, Medical Faculty; Heinrich-Heine University \\ Düsseldorf \\ ${ }^{3}$ Institute of Neuroscience and Medicine (INM-1), Research Center Jülich \\ ${ }^{4}$ Interdepartmental Neuroscience Program, Yale University \\ 5 Department of Radiology and Biomedical Imaging, Yale University \\ ${ }^{6}$ Department of Neurosurgery, Yale University \\ 7 Department of Electrical and Computer Engineering, ASTAR-NUS Clinical Imaging Research Centre, \\ Singapore Institute for Neurotechnology and Memory Networks Program, National University of Singapore, \\ Singapore \\ 8 Martinos Center for Biomedical Imaging, Massachusetts General Hospital, Harvard Medical School, \\ Charlestown, USA \\ ${ }^{9}$ Centre for Cognitive Neuroscience, Duke-NUS Graduate Medical School, Singapore
}

\section{ABSTRACT}

One of the most specific but also challenging properties of the brain is its topographic organization into distinct modules or cortical areas. In this paper, we first review the concept of topographic organization and its historical development. Next, we provide a critical discussion of the current definition of what constitutes a cortical area, why the concept has been so central to the field of neuroimaging and the challenges that arise from this view. A key aspect in this discussion is the issue of spatial scale and hierarchy in the brain. Focusing on in-vivo brain parcellation as a rapidly expanding field of research, we highlight potential limitations of the classical concept of cortical areas in the context of multi-modal parcellation and propose a revised interpretation of cortical areas building on the concept of neurobiological atoms that may be aggregated into larger units within and across modalities. We conclude by presenting an outlook on the implication of this revised concept for future mapping studies and raise some open questions in the context of brain parcellation. 


\section{The brain a topographically organized organ}

The brain is without question the most complex structure in the human body and we are still far from understanding its physiological functioning or pathology. In this context, one of the most distinct features of the brain in comparison to any other organ of the body is its topographic organization. This distinction and its implications become most evident when considering the effects of partial damage or removal. In organs like the liver, every part looks and acts in the same way and hence a resection of tissue only affects overall capacity, e.g., for metabolism, but not any specific function, e.g., bile production. In stark contrast, localized damage to a small part of the brain will result in very specific disturbances of isolated mental facilities, such as vision, movement or language (Rorden \& Karnath, 2004). In turn, however, overall function is usually not impaired following localized damage, i.e., while vision may be specifically affected, all other mental functions may be largely intact. Another important feature of this regional differentiation is the fact that the brain is realized on a common building plan. This renders the brain not only distinct from uniform organs but also from compartmentalized ones such as the kidneys, where different functions are realized in morphologically different sections. This topographic organization becomes most evident with respect to the cerebral cortex, which features a common building plan into six different layers of varying cell density (Amunts \& Zilles, 2015). In spite of the fact, that the entire cortex is made up from the same cell-types, however, variations from this common architectonic scaffold are also evident, as density, size and distribution of cells across the cortical layers may be observed. Likewise, the general principles of local and long-distance connectivity seem rather consistent across the brain, yet the origins and terminations of fiber bundles linking different parts show a highly differentiated pattern (Felleman \& van Essen, 1991). It may be noted, that topographic organization is not a specific feature of the human brain, but rather seems to have emerged very early in evolution and is shared by all vertebrates, despite vastly different instantiations and capacities (Krubitzer \& Kaas). A topographic specialization thus seems to represent a fundamental principle of brain function, rendering its investigation through what has now become known as brain mapping the key to understanding (human) brain organization.

\section{Historical development and conceptual changes}

One of the most curious facets in the history of human brain mapping is the fact that virtually all of its key concepts were anticipated by the 18th century phrenologists long before the emergence of neuroscience as the current biomedical discipline (Finger 2004). Evidently, their simplistic view of mental organs or facilities and the idea that these could be assessed by examining craniological features is so remote from the current scientific paradigm, that "neo-phrenology" has become a derogative term for over-simplified localization of cognitive functions. It is worth noting, though, that the works by the protagonists of that field, such as Johann Kaspar Lavater (1741-1801) and Franz Joseph Gall (1758-1828), actually spelled out most of the fundamental ideas that form the basis of human brain mapping and its clinical applications. Most importantly, they postulated that the brain was the physical basis of the mind and not a homogeneous organ. They hypothesized that the brain consisted of a topographically organized mosaic of individual modules supporting different functions and showing inter-individual variability that related to phenotypical features.

The first step towards establishing the topographic specificity of mental functions compatible with current scientific standards was taken in 1861 when Paul Broca described his famous patient suffering from non-fluent aphasia, but no other deficits. This condition had developed following a lesion in the left inferior frontal cortex. Broca's hypothesis that this specific deficit was caused by 
isolated damage to a specific part of the brain received heavy criticism from his peers supporting a holistic view on brain function (equipotentiality, pioneered by Lashley and Flourens). In the decades that followed, however, inferring the localization of cognitive functions through observation of the effects of localized lesions not only established itself as the primary approach towards understanding the organization of the human brain, but also shaped emerging concepts on brain organization. Most importantly, it fostered the notion of topographic specialization and structurefunction relationships that has remained largely intact until the most recent focus on brain connectomics (Sporns 2011).

One of the key developments that supported this localizing, topographic view of (human) brain organization was the emergence of microscopical anatomy in the late 19th and early 20th century. Using histological analysis of brain sections stained for cell bodies or myelin, researchers like Paul Flechsig, Cécile and Oskar Vogt, von Economo and Koskinas, and Korbinian Brodmann (for a detailed review, cf. Zilles \& Palomero-Gallagher, 2011) provided detailed descriptions of the microarchitecture of the human cerebral cortex. Histological analysis of stained, serially sectioned post-mortem brains provided the first opportunity to investigate the heterogeneity of microstructural features across the whole brain and hence provide complete maps of its differentiation. More specifically, cytoarchitectonic analysis is based on sections stained for cell bodies and assesses the relative width and cell-density of the six canonical layers of the (neo-) cortex, the distribution of different cell types and the size and arrangement of (in particular) pyramidal cells. In turn, myeloarchitectonic investigations are performed on sections stained for myelin and focus on the distribution of radial and tangential fibers within the cortex as well as the presence and location of myelin-dense bands. In these "classical" brain mapping studies, it was noted, that the cyto- or myeloarchitectonic characteristics of the cortex usually don't vary smoothly across the brain but tend to change abruptly at particular locations, which were considered to represent the borders between different cortical areas. This concept was most clearly advocated by the Vogts' based on the myeloarchitectonic work, who posited "haarscharfe Grenzen" (borders sharp as a hair) in the cerebral cortex, a feature that is also clearly expressed in the cytoarchitectonic work of their student Korbinian Brodmann. In turn, von Economo and Koskinas, while also following the general concept of abrupt changes between cortical fields, were more likely to also denote transition zones and local variations within a given cytoarchitectonic area.

Summarizing their results in a drawing showing the locations thereof on a picture of the brain, usually an idealized surface view, they quickly established the standard language we know today. Much like the political organization of, e.g., Europe, the topographic organization of the human brain can be represented by a map showing individual cortical areas of homogeneous architecture and the borders between them. And although the relation between these maps and the neuropsychological symptoms arising from localized lesions was rarely directly assessed at that time, the prevailing notion was that these distinct areas should also map to distinct functions. Owing to the refinements in the methods for mapping function (based on cognitive deficits following brain lesions) and architecture (based on histological examination), the next decades provided increasingly complex brain maps, defining smaller and smaller sub-areas.

In spite of this general trend, which may be considered ongoing if not accentuated with the advent of neuroimaging (Raichle, 2009), it is noteworthy that the compartmentalization of the brain has been challenged throughout its entire history. For example, shortly after the localization of language-relevant areas by Broca and Wernicke, Ludwig Lichtheim already proposed a model that highlighted the role of connections and interactions in the context of language production. In turn, while researchers such as Edith Gerhard and the Russian School provided increasingly detailed anatomical maps of the human brain, Gerhardt von Bonin and Percival Bailey proposed a map that considered almost the entire cortex as largely uniform, advocating a holistic view of brain organization (Bailey \& von Bonin, 1951). Yet, the prevailing view was that of topographic 
organization suggested by anatomical brain maps, localized lesions and physical white-matter dissection in post-mortem brains, even though the relationships among them remained opaque.

\section{New perspectives studying non-human primates}

While many early anatomical studies were already comparative in nature and features such as the cortical column were initially described in the cat (Mountcastle 1957), views on human brain organization were most profoundly influenced by the strong growth of physiological studies in nonhuman primates in the 1970s. Such studies entailed two substantial advantages that were critical for the conceptual development of brain mapping. First, studies in non-human primates enabled the use of invasive methods that to date must be considered the gold standard for the investigation of brain function, connectivity and structure. Invasive electrophysiological recordings provided for the first time spatially detailed information about neuronal response properties in the intact brain under a wide variety of experimental contexts (Hubel \& Wiesel, 1968). In turn, size and location of naturally occurring lesions in the human brain are strongly influenced by the vascular architecture and their effects can only be assessed following recovery of the patient, i.e., after substantial plasticity and adaptation has already happened (Mah et al., 2014). Single unit recordings and the measurement of local field potentials in monkeys thus provided an unprecedented view on local differentiation of brain function, confirming and extending previous theories on topographic organization (Levy \& Goldman-Rakic 2000). In addition, antero- and retrograde tracers that are absorbed by the neurons at the injection site and then moved towards either the soma (retrograde tracers) or the axon terminals (anterograde tracers) via axonal transport provided detailed information on the long-range connections between different brain regions (Rockland \& Pandya, 1979). These studies revealed that long-range connections follow a precise topography and specifically link individual areas across the brain. Finally, given that the animals needed to be sacrificed to verify the location of the recording sites and to reveal the transported tracers, histological investigations are readily performed using the same cyto- and myelo-architectonic approaches that formed the basis of classical human brain maps (van Essen et al., 1981).

The above naturally leads to the second critical advantage of such studies over the then (and one could argue currently) available methods for studying the human brain. In non-human primates, structure, function and connectivity of the brain may not only be assessed with a very high precision and specificity, but moreover all three aspects may be investigated within the same animal and hence directly compared to each other. That is, all three aspects of cortical differentiation were not only assessed with high fidelity but could moreover be spatially related to each at the millimeter-scale within the same individual. Throughout many of the seminal publications over the next decades, one of the most consistent observations was a good congruence between microanatomical borders, distinctions in connectivity patterns and variations in functional response properties. That is, if injection sites were at different sides of a cytoarchitectonic border, they usually differed in their projection patterns, if two locations showed different response properties, they were distinct in myelo-architectonic features and so on (cf. Burton et al., 1995 and Krubitzer et al., 1995).

This within-subject agreement of topographic differentiation across different features (at least within the sensory and motor cortices which were predominantly investigated) confirmed, albeit in different species, the prevailing notion that histologically defined areas in the human brain should correspond to functional modules whose damage would then lead to particular neuropsychological symptoms. The observations outlined above thus culminated in what is now considered the "classical" definition of a cortical area. 


\section{The concept of cortical areas}

In non-human primates, a cortical area is conventionally defined as a region of the cortex that may be distinguished from its neighbors by several criteria (for reviews see Felleman \& Van Essen 1991, Orban et al., 2004; Krubitzer \& Kaas 2005; Eickhoff \& Grefkes, 2011). First, a cortical area should show different global functional properties than the surrounding cortex. Second, it should be microstructurally distinct, i.e., differ in cyto- and myeloarchitecture. Third, it should feature a different pattern of inter-regional connectivity than its neighbors. Finally, given that most of the experimental work that has led to this conceptual definition of cortical areas has been performed in sensory cortices, in particular the visual system, the presence of a complete representational, e.g., retinotopic, map has likewise entered the definition of a cortical area. This definition not only reflects the historical development of concepts on topographic organization as outlined above, but has moreover been among the concepts most strongly shaping the emerging field of neuroimaging. Several aspects of the outlined definition are particularly noteworthy in the context of this review, most of which are attributable to the fact that the concept of cortical areas was primarily defined based on investigations of sensory cortices, in particular the visual system. Maybe the most obvious is the importance of topographic maps, which is indeed a hallmark of sensory areas to the extent that a double representation of a particular part of the body or visual field provides one of the strongest indications of the presence of an additional cortical area (Krubitzer et al., 1995, cf. Zeki et al., 1998). When moving away from early uni-modal cortices, however, such topical organizations become increasingly difficult to define, even though there is evidence for, e.g., retinotopic features in association cortex (Selemon \& Goldman-Rakic, 1985). Consequently, this criterion has been down-weighted when considering areas outside uni-modal sensory cortices.

Two implications of the classical definition of a cortical area had formative influences on the fields of neuroimaging and in-vivo brain parcellation (cf. Amunts et al., 2014). First, the classical definition of a cortical area inherently requires that within any single modality, i.e., structure, function and connectivity, there are clearly demarcated borders, rather than gradients or transition zones. In turn, within a particular area, this feature should be homogeneous. While both of these fundamental assumptions are rooted in the classical anatomical works by Brodmann or the Vogts, who advocated the concept of haarscharfe grenzen, i.e., borders sharp as a hair, they are actually far from trivial or undisputed. On the one hand, there is ample evidence that structural and functional features may be organized in gradients, in particular in the posterior parietal and prefrontal cortex (cf. Carmichael \& Price 1994 and references therein). On the other hand, other aspects of cortical organization such as the internal differentiation of early visual areas into blobs, stripes and ocular dominance columns (reviewed in Yoshioka et al., 1996) likewise challenge the assumption of homogeneous areas with sharp transitions of neurobiological features at their borders.

The second fundamental assumption is that a cortical area should be differentiated from its neighbors by all three aspects, i.e., structure, function and connectivity, with the respective borders from each criterion being in close agreement with each other. There is ample evidence from nonhuman primates demonstrating such agreement made possible through microscopic spatial resolution and the possibility to perform multi-modal comparisons of all three aspects in the same individual as described above. Nevertheless, there are also numerous instances in which a sharp change in one property is not mirrored in other aspects of cortical organization. One particularly 
interesting example of this situation, which is not confounded by different spatial scales, intersubject registration or blurring is provided by in-vitro multi-receptor autoradiography (Amunts \& Zilles, 2015). In this work, the binding sites for various receptors for classical neurotansmitters are marked on neighboring 20 micron thick sections of post-mortem brains using tritiated ligands. Looking at the early visual cortex, i.e., the part of the brain from which the concept of a cortical area was primarily derived, it may be noted, that in virtually all instances borders, evidenced by changes in mean receptor concentration or laminar pattern of receptor density, are closely matching the cytoarchitectonic borders between visual areas (Eickhoff et al., 2008). However, at the same time any particular border is usually only revealed by a subset of the investigated receptors (Figure 1). This duality of a close match between revealed borders but frequently absent transitions in some features has recently been mirrored by an integrated analysis of cortical myelination, functional activation properties and resting-state connectivity.

\section{---- Figure 1 about here please ---}

\section{Theoretical and practical relevance of the "cortical area" concept}

In spite of the conceptual limitations noted above, which were already known by the time that neuroimaging emerged and human brain mapping began to grow into the large and highly dynamic field it is now, the concept of a cortical area has a tremendous impact on our field. In fact, we would argue that the idea of a cortical area as a field that is distinguished from its neighbors by (sharp) changes in structure, function and connectivity, must be considered as the conceptual backdrop of most research in human brain mapping". But why has this concept caught on so well in neuroimaging and to date remained one of the core concepts for the analysis and description of brain organization?

The key appeal from both the theoretical and computational side is the idea of modular brain organization encoded by the term "cortical area", rather than the concept itself. The most important aspect here is that such modules or "cortical" areas may be regarded as units of coordinated information processing that act as a unified whole in terms of the sustained functions and in terms of interactions with other modules (Eickhoff \& Grefkes, 2011; Fox \& Friston 2012; Deco et al., 2015). This view not only accommodates the notion that the brain is topographically organized into distinct units, but also acknowledges the importance of specific interactions between them through the criterion of distinct connectivity (patterns). The idea of a "cortical area" thus provides the currently most widely accepted view of the brain as a highly-specifically interconnected network between specialized modules (Bullmore \& Sporns 2012, Park \& Friston 2013; Amunts et al., 2014).

Besides this conceptual relevance of defining what could be considered as the smallest meaningful building block (cf. next section), it is worth reviewing the concept of cortical areas or modules as the units of investigation. In particular, the resolution of current neuroimaging data (Uğurbil et al., 2013) is well coarser than the size of a cortical column (Horton \& Adams, 2005 but cf. Yacoub et al., 2008), but finer than the macroscopic neurobiological organization of the brain. Hence, the nominal dimensionality of 200,000 - 400,000 grey matter voxels (at a resolution of $1.5-2 \mathrm{~mm}$ isotropic voxel size and depending on the amount of included brainstem) is almost certainly not the one that would best represent the phenomenon of interest, i.e., cortical differentiation. Rather, it poses a substantial computational challenge, e.g., when trying to compute whole brain dense (voxel $x$ voxel) connectomes, leading to matrices that require too much memory for most standard 
computers (Loewe et al., 2015). Likewise, any multivariate analysis of voxel-wise data, in particular across a large number of subjects, will be inevitably challenging. Moreover, even if computationally tractable, analysis of neuroimaging data at the resolution of individual voxels is complicated by smoothing effects both from MR physics sources as well as from the reliance on the spatially broad BOLD effect (Ugurbil 2016).

Using cortical areas as a neurobiologically meaningful dimensionality reduction thus entails many advantages. First, working at the level of a cortical area, rather than at the voxel level greatly ameliorates the number of features and hence provides a much more efficient framework for, in particular large-sample, analysis. More importantly, however, it provides an a priori, biologically motivated approach to feature reduction, providing a potential solution to the important but ultimately open question of how to define nodes for connectivity analyses (cf. Zalesky et al., 2010, Caspers et al., 2013; Bellec et al., 2015; Thirion et al., 2014). That is, if a parcellation of the cortex is in line with the conceptual definition of a cortical area, these modules should each be homogeneous in, but distinct from, each other in terms of all measured or derived properties, e.g., volumetric measures or connectivity-profiles. Consequently, a parcellation into cortical areas promises to be optimal for the investigation of any sort of data. In contrast, any data driven dimensionality reduction (Craddock et al., 2013; Eickhoff et al., 2015) should be modality and potentially dataset specific. Moreover, given that cortical areas are supposed to reflect modules sustaining specific operations, there would be a natural link between the units of analysis and physiological as well as pathological function. Last but not least, the analysis of the structure, function and connectivity of cortical areas, rather than voxels or data-driven lower-dimensional representations, can provide a standard space that allows comparisons between studies while still allowing voxel-wise analyses, which could be allocated to the respective modules. In summary, it may hence not surprise that the concept of "cortical areas" has been a primary focus of neuroimaging throughout its existence.

\section{The issue of scale and granularity}

Importantly, it may be argued that cortical areas in the manner that they are usually defined by the aforementioned criteria in humans and experimental animals seem to provide a very useful intermediate level of detail. This may be best exemplified by juxtaposing areas such as "V4" (in the ventral visual cortex, cf. Zeki et al., 1998) to theoretically possible alternatives at different scales.

At the finer resolution, there is little doubt that the cortical column is the most basic unit of information processing and the fundamental architectonic building block of the brain (for a critical review of this assertion see Horton and Adams 2005). However, there are at least three major arguments against considering the cortical column as the fundamental unit of brain mapping. First, mapping the cortex at the columnar resolution is currently impossible given that the primate brain contains many millions of cortical columns. Second, the in-vivo investigation of individual cortical columns is difficult with classical invasive approaches or current neuroimaging (though there may be hope cf. Ugurbil 2016; Yacoub et al., 2008; Grinvald et al., 2000). Third and probably most importantly, while cortical columns in sensory cortex appear to have unified tuning properties, the level of functional specialization that may be ascribed to a particular cortical column is well below the "behavioral" resolution, i.e., the level of abstraction needed to describe brain functions at a behavioral level. In other words, only the integrated processing of multiple cortical columns may yield a neurobiological function that relates to a mental process. 
In turn, a broader resolution may be provided by macro-anatomical divisions, such as the (posterior) fusiform gyrus (Desikan et al., 2006) or by reference to coarse maps of the brain such as provided by Brodmann's 1909 book. Neither of them, however, are particularly useful for the delineation of a cortical module that serves as the basic unit of processing at a behaviorally accessible level. Most importantly, both of these descriptors are too broad to relate to behavioral operations in a meaningful fashion. Rather, they appear to harbor multiple modules that are recruited by different tasks and are connected to different large-scale networks. Considering, e.g., the entire supramarginal gyrus as a single module will lead to a very heterogeneous association with functions or deficits behavioral profile, and an undifferentiated profile of connectivity with other areas (cf. Caspers et al., 2011). This is most evident in cases such as the temporo-parietal junction in which very distinct sub-regions are present, which would be smeared together in the case of a broad anatomical definition (Mars et al., 2012; Bzdok et al., 2013). In other words, considering large (macro-anatomical) delineations as the individual nodes of brain organization will most likely fail to provide any relevant insight into the organization of functional networks (Zalesky et al., 2010). In this context, one of the great ironies of the field is that only the coarsest of all classical (cytoarchitectonic) brain maps, the one by Korbinian Brodmann was translated into English early and later used by Talairach and Tournoux (1988). While even these authors themselves note, the indication of the areas in their atlas is a rough estimate based on Brodmann's schematic drawing and was only meant for illustrative purposes, multiple (again extrapolated) versions of the Talairach atlas (e.g., Lancaster et al., 2000) are still widely used in use today. Thus, while many much more detailed classical and modern histological maps of the brain are available, the subjective guess of where the areas of the coarsest available atlas should be located in 3D space is widely considered as the standard reference of the brain. Obviously, however, these areas are much coarser than any functional differentiation, leading to two scenarios. In some cases, a host of different functions is assigned to the same Brodmann area (BA), as evident, e.g., for BA 9 or BA 39. In other cases, the broad labels of the Talairach / Brodmann atlas are unsystematically mixed with labels originating from primate research, which may be far from congruent, e.g., V3 (BA 19, cf. Rottschy et al., 2007) or PMd (BA 6, cf. Genon et al., 2016). While they may provide some common language of where a finding is located, it becomes clear that, like cortical columns, such large anatomical regions do not seem to reflect the true modular organization of the brain.

These considerations evidently lead to the crucial question of the "right" granularity from the neurobiological point of view. Here it is important to remember, that the brain is a hierarchically organized system in which individual elements (at the lowest level most likely the cortical column) are working together in a coordinated manner to implement a particular process. Neighboring sets of columns perform a similar process that is used in concert. The ensuing ensemble is closely linked with others that usually cooperate in most settings and ultimately with yet other regions that may be considered part of a limited number of canonical large scale networks (Scannell et al., 1999; Ross, 2000; Sporns et al., 2004; Fox et al., 2005; Kaiser 2011; Yeo et al., 2011; Power et al., 2011; Fox \& Friston 2012). Consequently, neither the fact that there are several million cortical columns nor the answer that there are 5-15 large scale networks provides incorrect or biologically meaningless answers to how many modules comprise the brain.

Nevertheless, in many cases the actual unit of interest should be what we will call neurobiological atoms. We would conceptualize them as modules that perform (only) one specific operation (independently of how this can be mapped to psychological terms) and behave as a unit in all relevant neurobiological features (that may yet need to be defined), while being distinct from all other units, even those with which they cooperate. We note, that this largely reproduces the definition of a cortical area, but deviates from the notion of spatial homogeneity and functional specificity. This reformulation has particular relevance in topographically organized regions, given 
that, e.g., the hand representation of the primary motor cortex is actually very distinct from that of the face, rendering them potentially different atoms as demonstrated in recent mapping studies.

One question that remains open for the time being, though, is the scale of these neurobiological atoms and the extent to which this scale is homogeneous across the brain. Addressing this question in a manner that is unbiased by the effective resolution of the applied imaging data or constrained by the computational approach remains one of the biggest challenges in understanding the topographic organization of the human brain as a very specifically connected network of specialized modules.

\section{Challenges in mapping cortical areas by human neuroimaging}

As argued above, the concept of a cortical area must be considered fundamental to most research in human brain mapping. Nevertheless it must also be noted, that its application in the context of current (human) brain imaging requires at least two extrapolations.

The first pertains to the fact that virtually all direct experimental evidence supporting the notion of a cortical area as a unit of distinct (micro-) structure, function and connectivity has been derived from non-human mammals (including in particular primates) rather than humans. In this context, we would argue that the neurobiological differences between our species and other primates at the fundamental organizational level should be small enough as to not warrant major concern about the validity of the concept of a cortical area in humans. However, what is strikingly different between monkeys and humans is our ability to examine this question experimentally. Whereas in monkeys, most methods provide highly specific, direct assessments, e.g., axonal connectivity or response properties (Disbrow et al., 2003), human neuroimaging relies on indirect measures yielding modeled rather than observed results (for an extended discussion and the role of monkey imaging, see Orban et al., 2004). In contrast to the situation in research animals, integration of invivo measurements and histological assessments of the human brain are not possible, introducing a fundamental gap between these two sources of information, which can only be bridged by between-subject comparisons (see, e.g., Wilms et al., 2005; Eickhoff et al., 2008). While this represents a major drawback to the within-subject comparison in monkeys, it may also be noted, that single-subject neuroimaging data tends to be rather noisy (often because insufficient data is collected), rendering borders revealed in an individual subject susceptible to artifacts and motivates group analyses, which are the standard in human brain mapping. Together with the intrinsic smoothness of neuroimaging data and the still limited capacity to overcome inter-subject variability, such group analyses are necessarily at a different level of spatial precision relative to what is possible in non-human primates. Consequently, we would argue that any evidence for convergence between different modalities and ensuing brain maps should not be strongly interpreted as direct experimental confirmation of structure-function relationships, although new high-quality within-subject data from the Human Connectome Project has certainly shifted the reference in that perspective.

In turn, one of the biggest advantages of neuroimaging relative to invasive methods is that they provide quantitative whole-brain maps of cortical features, be it activations evoked by experimental tasks, information on structural and functional connectivity or (coarse) anatomical information such as myelination or grey matter thickness (Amunts et al., 2014; Glasser et al., 2016). This aspect is particularly relevant in combination with the notion that cortical areas are supposed to feature sharp boundaries, as e.g., evidenced by the relatively rapid transition of cyto-, myelo- or chemoarchitectonic features (Zilles \& Palomero-Gallagher 2001; Eickhoff et al., 2008; Henssen et 
al., 2016). Using invasive recordings, however, functional response properties may only be tested along the electrode path. While every recording provides spatially precise information on the response to a set of task-features, the sampling across the cortex is thus very limited (Krubitzer et al., 1995) as is the set of task-features that can be tested. The same issue applies to injection sites for tracing studies (Burton et al., 1995). Hence, notwithstanding their superior specificity and precision, invasive methods suffer from limited sampling in both spatial and functional domains.

The second extrapolation pertains to the difficulties of applying a concept that was mainly developed in sensory cortices to higher order association areas, such as the parietal or in particular prefrontal cortex. Several aspects contribute to this predicament. First, as already noted, concepts of topographic representation of the sensory space are not applicable in brain regions processing more abstract information. There is also evidence suggesting that transferring the remaining criteria may likewise come with some caveats. For example, connectivity patterns within uni-modal sensory cortices are not only topographically organized but also follow rather well described logic and entail mainly short-range, i.e., within system connections (cf. Fellemann \& van Essen, 1991; Markov et al., 2012). In turn, higher association regions in the prefrontal, parietal or temporal cortex are marked by widespread or diffuse connectivity patterns indicative of their integrative role (cf. Carmichael \& Price, 1994; Wise et al., 1997). Importantly, this is not to say, that neighboring areas within these regions may not be differentiated by their connectivity pattern, as this has been shown for cytoarchitecture or connectivity-based parcellations (for a comprehensive series of studies on the fronto-polar cortex cf. Bludau et al., 2013; Ray et al., 2015; Bludau et al., 2016). However, it still remains to be seen, whether the same degree of mechanistic understanding as for sensory cortices is also attainable for higher associative areas.

The notion that cortical areas should also show differentiated function and dysfunction is at the core of the idea of a cortical area and ultimately also of highest interest in terms of understanding brain organization and pathology (Eickhoff \& Etkin, 2016, Fox \& Friston, 2012; Amunts et al., 2014). In this context, we note that theoretically functional differentiation between cortical areas needs to follow from differentiations in structure and connectivity. That is, the specific function of a particular patch of the cortex can only arise from two aspects, namely its architecture and connectivity. In other words, what a particular region of the brain is doing should be determined by the local infrastructure for computations (reflected among other features by cyto-, myelo- or receptor-architecture) and its pattern of inputs and outputs (although it remains an open question, how these are best captured). Unfortunately, however, this deterministic relationship between structure and connectivity on the one hand and function on the other seems experimentally inaccessible in the foreseeable future, given that we do not yet know, which features define function in which way and how to measure them.

This leads us to probably the most critical challenge when mapping "higher" cortical areas, namely how to define function in these regions. The description and categorization of mental functions has a rich tradition in experimental psychology (for recent approaches and a conceptual overview, cf. Poldrack et al., 2011 or Turner \& Laird, 2012). Yet, most of these are quite dependent on the actual theory and few aspects are universally accepted. One example (Miyake et al., 2000; Alvarez \& Emory 2006) would be the nature of executive functions (which may be seen as a unitary or fragmented construct), how to probe them (given that different tasks clearly tap into several theoretical factors) and their relationship to other functions such as working-memory (which may be considered part of the executive system or not). Moreover, the psychological descriptions of high-level processes are often rather vague attempts to verbalize abstract processes. For example, self-control, task-set implementation, schema energization and monitoring, awareness, salience detection, outcome prediction and evaluation, contextual updating, alertness and arousal 
regulation as well as effort investment have all been used to describe a process necessary to complete effortful but goal-directed tasks (Langner \& Eickhoff, 2013). This evidently questions, whether such vague concepts can be used to functionally differentiate neighboring regions, e.g., in the anterior prefrontal cortex. Finally, the relationship between psychological concepts and the neurobiological differentiation of function must be considered a largely open question. That is, whether the atoms of neurobiological computation are congruent with the atoms of mental functions hypothesized by experimental psychologists needs to be considered with skepticism.

\section{Cortical areas: impact and critique}

The idea of a cortical area has a longstanding tradition in the field of (human) neuroscience and was most explicitly defined in the late 20th century as a field that is distinguishable from surrounding ones by differences in (micro-) structure, functional properties and connectivity patterns. It has come particularly into focus with the more recent advent of in-vivo brain parcellation methods (Eickhoff et al., 2015; Glasser et al., 2016; Yeo et al., 2011; Clos et al., 2013; Craddock et al., 2013; Shen et al., 2013; Bellec et al., 2015; Thirion et al., 2014; Behrens et al., 2003; Fan et al., 2016). We here argued, that the relevance of cortical areas arises particularly from their potential to act as a biologically meaningful dimensionality reduction into homogeneous modules at a behaviorally relevant scale.

As we will outline in more detail below, using this teleological definition the number and location of cortical areas in the human brain needs to be considered as yet unknown. In addition, it must be remembered that the three aspects structure, function, and connectivity may be broken down into many individual features (Yeo \& Eickhoff, 2016). Some features are evident and experimentally accessible (e.g., distribution of different receptors, functional or structural connectivity), while others are still poorly defined (e.g., function in "higher" multi-modal associative areas). From these considerations, several important consequences may be derived. First, cortical areas deemed homogeneous in some (combination of) modalities may actually show intra-areal heterogeneity in a new one. Second, while different features are usually in good spatial agreement regarding the location of revealed borders, probably no border is revealed by all features. Finally, it must also be acknowledged, that the entire concept of cortical areas is build on the assumption of sharp transitions and hence might poorly accommodate gradients and smooth transition, which may also play a role in cortical organization, even though most evidence clearly favors definite and well localized changes in each feature of structure, function and connectivity.

\section{A conceptual re-evaluation of cortical differentiation}

The key to reframing the concept of a cortical area, in particular in the context of current approaches to human brain mapping and parcellation, is the notion that the three classical aspects of brain organization, i.e., structure, function and connectivity, are rather coarse collections of individual features. Considering the current repertoire of histological and in-vivo approaches to provide location specific information about the human brain however, the amount of possible features has now grown to an extent that the use of these major categories must become questionable. Moreover, it must be noted, that current developments have made the boundaries between structure, function and connectivity rather fuzzy. For example, it may be argued that receptor-architectonics (Zilles et al., 2015) could either be classified as structure (in line with other histological approaches) or function (given the direct relevance of receptors and their regionally 
specific balance to signal processing). Likewise, meta-analytic co-activation mapping examining the likelihood of joint recruitment with a region of interest across the whole-brain (Fox et al., 2014) may be considered a measure of function or connectivity. In other words, while the conventional thinking about structure (historically referring to micro-architectonic features), function (implying a definable response-profile) and connectivity (classically defined by invasive tracing yielding axonal connections) has its value from the conceptual point of view, it may also provide a restraining historical background for the current possibilities to describe (human) brain organization.

We would hence propose to conceptualize regional differentiation of the brain and in particular the cerebral cortex by location-specific multivariate feature vectors (cf. Figure 2 for an illustration how such features may look like for a particular brain region). That is, each cortical position would be associated with a potentially large number of features describing a multitude of dimensions. These features may natively live on different spatial scales owing to the differences in resolution between, e.g., microscopic examination, structural and functional MRI, and PET imaging, resulting in up- or downsampling when moving to a specific resolution. Here we note, that there probably is not a single resolution that may be naturally preferred but rather the optimal resolution should depend on the available data and the question at hand, with a lower bound being provided by the resolution of a cortical column.

\section{---- Figure 2 about here please ---}

There is no doubt, that many of these features will be variants of the three classical categories, though most likely adapted to the current state of neuroimaging and human brain mapping. For example, we expect histological descriptions of cortical differentiation such as cyto-, myelo- and receptor-architecture to play a major role in future endeavors to brain parcellation (Amunts \& Zilles, 2015). But most likely, these will be complemented by more recent in-vivo methods that may serve as architectonic proxies, such as mapping (relative) myelin content (Stikov et al., 2015; cf. Glasser et al., 2016). In this context, post-mortem MRI that can be cross-validated against histology in the same brain (Eickhoff et al., 2005b; Large et al., 2016; Foxley et al., 2014; Sampaio-Baptista et al., 2013) will provide a particularly important bridge between the different scales and may provide important insight into the biological nature of MR-based architectonic features. We would furthermore expect that the most critical contribution in terms of in-vivo structural imaging will come from those approaches that provide additional information not available from classical histological methods. One example in the context of brain structure would be structural covariance patterns across large populations (which seems to be distinct from both functional connectivity and anatomical connections, cf. Reid et al., 2016). Even though the relative contribution of genetic and environmental contributions to structural covariance are still under debate, it has been shown that this approach exhibits spatially distinct patterns that may be used for brain parcellation (Kelly et al., 2012). Extending this thought, patterns of co-development during childhood, co-involution in the context of aging and co-affection by neurological or psychiatric disorders (for an example in development cf. Zielinski et al., 2010, for a clinical example see Zhou et al., 2012) may provide a vast feature-space, even though much more research into these developing fields of brain mapping are necessary.

Notably, these examples also highlight the often-neglected scale of inter-subject averaging along features that may be placed in different extremes (cf. more detailed discussion in Amunts et al., 2014). On one extreme, features such as orientation columns in the visual cortex may be clearly identified in individual subjects but cannot be spatially aggregated across subjects given that their 
arrangement and number is highly variable (Yacoub et al., 2008). Here information needs to be extracted, e.g., not orientation columns themselves but their presence may represent a relevant feature. On the other, features such as structural covariance patterns or task-based co-activation patterns across a wide range of neuroimaging experiments may only be identified at the group level (Reid et al., 2017). In between, many features such as structural connectivity patterns might bridge the different scales of agglomeration or averaging.

In summary, a detailed multivariate description of each brain location (voxel or surface vertex) may provide a much more complete representation of topographic organization of neurobiological features and hence brain organization. It also holds great flexibility towards incorporating new, developing and yet unforeseen characteristics, given the non-static nature of the description. In turn, a brain atlas consisting of a multivariate feature vector per location also requires few assumptions other than the possibility of sufficient registration between individuals. We note, however, that the latter assumption may be critical (and may provide an upper bound on resolution), given that any registration needs itself to be driven by some set of recorded features and the choice of those features that drive the image registration may in turn bias the ensuing multivariate description (Robinson et al., 2014; Mangin et al., 2016). Moreover, the proposed concept has, at this stage, no real power for labeling a particular location as a particular cortical area or for providing a biologically meaningful data compression, i.e., the problem of "where are the cortical modules" remains to be addressed as outlined below.

\section{A revised concept of cortical areas}

At the theoretical limit of meaningful resolution and considering all possible features, each cortical location should be associated with a unique multivariate profile reflecting its specific neurobiology. Evidently, this conjecture depends on the effective resolution of the assessed features, which in turn will be determined by the native resolution of the data, the variance introduced by spatial normalization and last but not least explicit smoothing. However, given a sufficiently large and diverse set of features, we would expect that even in the light of the limited effective resolution of current methods, almost no pair of locations will have an identical set of multivariate features.

This contemplation is critical to the definition of cortical areas from location-specific multivariate profiles, given that it challenges the current idea of an area being an entity that is not only distinct from its neighbors in all aspects but also internally homogeneous. While this traditional view seems hardly compatible with the concept of location specific profiles as outlined in the last sections of this paper, this does not imply that the concept of a cortical area representing a fundamental module of topographic organization needs to be abandoned. Rather, it calls for a revised concept that accommodates the cumulative evidence on regional differentiation reviewed here. One of the most critical aspects in this context is scale, both spatially and in terms of neurobiological similarity. We argue that the crucial contribution that has been made by the notion of cortical areas, i.e., the definition of distinct modules, requires a granularity at which the differences between areas remain interpretable. Meaningful modules thus need to be of a sufficient size in order to represent a local summary of data and, potentially even more importantly, the differences between them need to be substantial enough to warrant a distinction. We acknowledge that the latter aspect may not be too easy to address quantitatively, given that the brain most likely follows a hierarchical organization yielding several potential cut-off points, each of which may define a relevant level of differentiation.

The existence of these potential cut-off points as opposed to a continuous scale of dissimilarity seems likely based on the cumulative evidence from more than 100 years of human brain 
mapping. Not only is there clear evidence for sharp transitions, i.e., borders in virtually any feature that has been studied up to now, but also it has been shown that borders revealed by different aspects usually co-localize very well. We would hence posit that each individual location should most likely show some differentiation from its neighbors in a multivariate feature space. At some points, however, adjacent locations will show more marked differences in several, though not necessarily all, features, indicating a cortical border at this position. That is, cortical borders exhibit more pronounced (within feature) and consistent (across features) differences in neurobiological properties than other locations. Moreover it stands to reason that different borders show a varying level of distinctiveness, corresponding to the hierarchical nature of cortical borders, ranging from those that may be regarded as too subtle to be meaningful at a given resolution to massive changes in virtually all features, e.g., between the SMA and pre-SMA. Hence, the distribution of neurobiological differences between neighboring locations should be right-tailed with distinct peaks at higher distances, indicating these borders at different levels of hierarchy (Figure 3).

\section{---- Figure 3 about here please ---}

Before considering how current approaches to brain parcellation based on neuroimaging fit into this concept and outlining potential future directions of the field, we would like to make three important notes. First, the idea of a complete feature space is primarily a conceptual one and probably not practically realizable, given the evolving methods for characterizing the neurobiology of the brain at different spatial, temporal and conceptual levels. The considerations above thus describe an idealized case in which all information has been obtained with minimal redundancy. Second, it stands to reason, that many features will be tightly related to each other, but unfortunately these relationship are far from understood even for established methods. This not only raises the need for more research on the convergence or divergence of neurobiological measures of brain structure, function and connectivity, but may also provide challenges for brain parcellation given that redundancy could be misinterpreted as convergent evidence. Finally, given that we can expect a continued expansion of our repertoire to characterize the human brain and our knowledge on the neurobiological underpinnings of these measurements, we would consider human brain mapping as a dynamic process. That is, for the foreseeable future, there will most likely not one final map of the human brain, but rather conceptual and technical refinement of cortical parcellation will remain a vital part of the brain research.

\section{Neuroimaging based brain parcellation: a rapidly growing field}

For many years, neuroimaging has primarily focused on either describing the characteristics of a priori defined regions of interest or, as the most common application, identifying voxels that are modulated by some experimental manipulation (usually, different task conditions) or that co-vary across individuals as a function of an external covariate (such as disease status or cognitive phenotypes). In turn, brain parcellation through the delineation of cortical areas and their borders remained the domain of histological work as represented by the JuBrain cytoarchitectonic atlas (Amunts \& Zilles, 2015), which in turn is closely integrated with neuroimaging analysis platforms (Eickhoff et al., 2005). Work integrating information about cytoarchitectonic areas with in-vivo imaging findings provided important support for the notion of cortical areas by repeatedly demonstrating that functional findings relate to histological subdivisions of the cortex (e.g., Wu et al., 2009; Wilms et al., 2005). Initiating the field of in-vivo brain parcellation, Behrens and colleagues (2003) showed, that by computing connectivity profiles for each voxel from diffusion weighted imaging and then clustering these voxels based on the similarity in connectivity patterns, 
they could recover different established thalamic nuclei or the distinction between SMA and preSMA (Johansen-Berg et al., 2004). Yet, for quite a few years, in-vivo brain parcellation based on neuroimaging data remained a rather niche field. This status underwent a massive change over the last half decade, during which in-vivo brain parcellation based on connectivity-information has developed into a burgeoning field as illustrated by figure 4 .

\section{---- Figure 4 about here please ---}

In this context, it is interesting to note, that while observer-independent analysis of cytoarchitectonic profiles has been around for almost two decades (cf. review in Amunts \& Zilles, 2015), this quantitative aspect of brain mapping has been critical to in-vivo brain mapping. In particular, virtually all published (in-vivo) brain parcellations are fundamentally based on either clustering locations (voxels) based on their neurobiological features, i.e., following a global approach comparing all voxels at the same time, or on comparing the similarities between features of neighboring voxels or vertices in order to detect those locations where the profile changes most pronounced, i.e., taking a more local, border-detecting approach (for a more detailed overview and methodological discussion, cf. Eickhoff et al., 2015; Glasser et al., 2016; Yeo \& Eickhoff, 2016; Bellec et al., 2015; Thirion et al., 2014). As for "classical", histological approaches, however, finding homogeneous regions or detecting sharp transitions constitute the conceptual basis of virtually all current brain parcellation approaches. In turn, methods allowing for smooth gradients and transitions are rare (cf. Figure 5). However, recent applications of mixed-membership clustering algorithms, allowing a region to participate in multiple clusters, can potentially address these issues (Yeo et al., 2014; 2015; Moyer et al., 2015; Najafi et al., 2016).

\section{---- Figure 5 about here please ---}

Several factors contributed to the rapid expansion of connectivity-based parcellation. From the supply perspective, large-scale, high-quality neuroimaging data has become much more readily available over the last years. This initially happened through bottom-up efforts such as the 1000Functional connectomes project pooling individual smaller datasets (Biswal et al., 2010). Later the pace was accelerated through larger projects focused on data collection and dissemination such as the NKI "Rockland" sample of NYU (Nooner et al., 2012) and the Human Connectome Project (van Essen et al., 2013), which has pushed the boundaries of MRI data in terms of resolution and quality (Ugurbil et al., 2013). The fact that high-quality imaging data is now openly available to the community has triggered a demographic shift in the neuroimaging community. Traditionally the field was dominated by medical and, to some degree, psychology departments, i.e., those that had best access to scanning facilities. The availability of large datasets, however, has now open the field to many individuals and labs from fields that traditionally had a focus on bigdata but no access to scanning facilities, such as engineering, computer science and physics. The ensuing surge of interest in, and knowledge of, multivariate big-data analytics has helped to spur the growth of brain parcellation from two sides. On the one hand, clustering, multivariate distance computation and dimensionality reduction have become much more commonly employed techniques in neuroimaging, leading to a shift away from univariate approaches that dominated neuroimaging in the first two decades (e.g., Mclntosh \& Mišić 2013; Shehzad et al., 2014; Bludau et al., 2016). On the other hand, research focusing on graph-theoretical connectome analysis, time-varying connectivity patterns, or the classification and prediction of clinical states and other phenotypical variables all urgently require a dimensionality reduction from the $>200,000$ individual voxels acquired in standard MRI protocols (cf. Zalesky et al., 2010, Bellec et al., 2015; Thirion et al., 2014, Rosenberg et al., 2016a,b). Thus, the supply of data, the demand for, biologically 
meaningful data compression along with the shift in culture towards multivariate big-data analytics, have combined to fuel the rapid expansion of neuroimaging based human brain parcellation.

It is interesting to note that the field is quite evenly split between studies pursuing whole-brain parcellations and those that take a more regional approach by mapping a particular part of the brain. The former provide a complete map at the expense of providing rather limited details on the individual modules (Gordon et al., 2016; Shen et al., 2013; Craddock et al., 2013; Yeo et al., 2011; but cf. Glasser et al., 2016 and Fan et al., 2016 for examples with relatively detailed characterization). The latter allowing much more detailed characterization and interpretation but coming with the drawback of requiring an a priori region of interest (Muhle-Karbe et al., 2016; Bzdok et al., 2015; Sallet et al., 2013; Mars et al., 2012; Thiebaut de Schotten et al., 2016). In contrast to this parity, in-vivo brain parcellation has been dominated by uni-modal parcellation studies, i.e., investigations that only consider one particular feature of brain organization, most frequently resting-state functional connectivity or structural connectivity as approximated by tractography on diffusion weighted imaging data. Studies that use alternative information for the additional characterization of the modules derived from (uni-modal) parcellation are rare (e.g., Fan et al., 2016; Cieslik et al., 2013), those that perform parallel parcellation of a given region using more than one feature of brain organization (usually connectivity) even more so (e.g., Wang et al., 2015; Genon et al., 2016). Finally, only a single study has up to now proposed a whole-brain parcellation by jointly considering multiple features that span the three major categories of structure, function and connectivity (Glasser et al., 2016).

\section{Uni-modal brain parcellation in the revised concept of cortical areas}

In the following, uni-modal refers to parcellation studies using a single feature to identify distinct modules either within a predefined region of interest or across the entire brain. We note, that this may represent some deviation from alternative uses of the term "modality", referring for example to the distinction between structure, function and connectivity, or to a particular imaging method such as MRI as opposed to for example histology. Several features have been used repeatedly for unimodal brain parcellation with some interesting divergence between whole-brain parcellations and regional studies. With respect to the former, resting-state fMRI connectivity data is clearly the dominant data source (Yeo et al., 2011; Shen et al., 2013; Craddock et al., 2013; Bellec et al., 2015), due to the fact that it provides functional connectivity information for every voxel in the whole brain. Additionally, the smooth and symmetric nature of this feature lends itself to the identification of larger systems across the whole brain, and is not limited to only regional differentiation. In turn, regional in-vivo investigations have most frequently been based on probabilistic diffusion tractography as a proxy for anatomical connectivity (Cerlani et al., 2016, Wang et al., 2016) although recent work has raised concerns about the problem of false tracks recently in the approach (Maier-Hein et al., 2016; Donahue et al., 2016). Meta-analytic coactivation mapping has also been used for this purpose (Bzdok et al., 2013b; Pauli et al., 2016), while resting-state analyses are less common. In addition, cytoarchitectonic mapping in serial histological sections likewise represents a uni-modal approach to mapping the human brain. Each of these approaches evidently has its own advantages and shortcomings. Most importantly, all methods apart from histological investigation depend on data processing and modelling, rendering the actual features more indirect measures as compared to the directly observable distribution of cell bodies. Structural imaging at the macroscopic scale, i.e., investigations of local volume, cortical thickness or relative myelin content, in turn most likely reflects a compound measure lumping together several neurobiological features at the histological scale (Eickhoff et al., 2005b). Finally, mapping based on connectivity features provides an important window into the network level 
integration of a particular location, but no current technique is optimally suited to reveal a ground truth on brain "connectivity". As noted above, diffusion-weighted imaging is closest to the notion of regions being (anatomically) connected to each other, but it may be questioned, how faithful tractograms reflect the underlying wiring pattern (Maier-Hein et al., 2016; Donahue et al., 2016). Resting-state imaging allows to characterize functional integration in distributed networks, but the rather prominent effects of data processing on the ensuing has lately gotten under quite some scrutiny (Power et al., 2015; Satterthwaite et et al., 2013; Varikuti et al., 2016). Finally, task-based co-activations allow to probe connectivity during more controlled settings and also show a intriguing convergence with resting-state findings (Smith et al., 2009; Reid et al., 2016), but are intrinsically limited by the nature of paradigms that may be performed in the scanner environment.

How do uni-modal approaches fit into the conceptual framework above based on of locationspecific multivariate profiles? Obviously, and independent of whether mapping is performed by clustering or boundary detection, such uni-modal parcellation will yield modules that are homogeneous with respect to the assessed feature and differ from each other with respect to it. In other words, the modules obtained from a uni-modal parcellation may be considered compressed representations of the data available for that particular feature at a given granularity. The latter aspect is important, as within every feature, there will be subtle differences between any neighboring pair of locations, more pronounced ones indicating putative cortical areal boundaries and finally massive changes at places where large-scale networks abut. Hence, whether userdefined or embedded in the algorithm, each parcellation provides a particular level of granularity. It may be noted, that even in the case of automatically chosen boundary criteria or cluster numbers, this choice is never entirely objective as the implementation of, e.g., evaluation metrics, threshold sensitivity or penalties, represents a priori choices. Consequently, parcellations using a single feature summarize the overall distribution of this feature by a number of sets (modules) that are distinct but internally homogeneous. Consequently, uni-modal parcellation can be primarily regarded as biologically informed data compression that may be highly useful for analyzing (new) data on that feature. For example, a robust parcellation of the brain based on resting-state connectivity will provide a priori information that allows the investigation of functional connectivity in another group of subjects at a computationally more tractable and presumably behaviorally relevant at the resolution of parcels rather than individual voxels or vertices (Bellec et al., 2010; Gordon et al., 2016; Finn et al., 2015, Finn et al., 2016).

In that context, we would also like to point out, that spatial contingency of the identified modules is an optional requirement, although it is enforced in most approaches. This is particularly relevant for cross-correlational methods such as resting-state functional connectivity, in which highly correlated time-courses across several (spatially separate) parts of the brain may result in their attribution to a single module if no continuity constraint is used (c.f. ICA decomposition methods). Thinking of unimodal parcellations as data compression, this behavior is perfectly reasonable as indeed the data is ideally represented in such manner. In turn, it also highlights why parcellations based on a single aspect do not necessarily provide information about cortical areas in the common interpretation of this term.

\section{Transferability of parcellations}

This is not to say that brain parcellation based on a single feature, be it cytoarchitecture (Henssen et al., 2016; Bludau et al., 2013), resting-state connectivity (Yeo et al., 2011; Shen et al., 2013; Bellec et al., 2010; Gordon et al., 2016) or grey matter volume (Sotiras et al., 2015; Evans 2013), may not approximate cortical areas defined from a multivariate feature space. As we have outlined 
above and in congruence with classical definitions, one of the defining features of cortical areas is that multiple features change conspicuously at their borders. In other words, borders defined by any given modality should coincide well above chance with borders defined by other features. One implication of this conjecture is that parcellations based on a single aspect of brain organization should provide modules that provide significantly better representations of other aspects (in terms of within-module homogeneity and module distinctiveness) than random clusters. That is, modules obtained from parcellation of the brain based on, e.g., resting-state functional connectivity should be, on average, internally more homogeneous and more distinct from each other in terms of cytoarchitecture or structural connectivity than random parcellations. The reason is that at least a certain proportion of the borders in the latter modality match those identified in the former, i.e., there is at least a partial recovery of the heterogeneity across the cortex of any other aspect. Nevertheless, it needs to be remembered, that at the same time a certain number of borders that would have been revealed by another aspect of brain organization are missed by any given feature. That is, congruence often is far from perfect (cf. Glasser et al., 2016 for an illustration that borders clearly present in one modality may be missing in another). Thus, any given feature might subdivide a homogeneous expression of another one. One interesting illustration of this situation is provided by Gordon et al. (2016) showing that resting-state homogeneity within cytoarchitectonically defined areas from the JuBrain atlas is higher than chance but lower than in resting-state defined clusters. Consequently, we would argue that parcellations based on a single modality will transfer to other ones, i.e., provide relevant information also on the spatial heterogeneity of other features, but not approach the quality of compressed representation obtained by a parcellation derived from that other feature itself.

One of the most interesting, and ultimately open, questions in that context is, whether some features provide parcellations that are more transferrable than others. This is basically equivalent to asking, whether the borders revealed by some features are more likely to coincide with those revealed by (the majority of) the other features. For example, it could be argued that cytoarchitectonic borders, reflecting a very prominent and directly observable rather than modelled feature of brain heterogeneity may represent locations at which (most) other features also agree (Caspers et al., 2013; Palomero-Gallagher et al., 2015). This is supported by several studies showing that in-vivo approaches to brain parcellation tend to confirm cytoarchitectonic borders. However, it must also be noted, that a lot of studies have already revealed that parcellations, e.g., based on connectivity or chemoarchitecture, may further subdivide cytoarchitectonic areas (for an illustration of this situation in Broca's area, see Amunts et al., 2010 and Clos et al., 2013). We consider this question about the "quality" of the information provided by any given feature, defined as its ability to detect borders that match those in other features and hence its transferability to other modalities, as one of the most important ones for future research. This is particularly true, given that little is yet known about the relationships between different measures of brain organization, i.e., structural, functional and connectional features, and their spatial patterns.

\section{Multi-modal brain parcellation}

Given that cortical areas should reflect modules whose borders show sharp changes across multiple, though not necessarily all, features, it stands to reason that any parcellation based on multiple features should provide a better approximation of the "true" biological heterogeneity of the cerebral cortex. This assumption is in line with the traditional definition of cortical areas and concepts of regional differentiation. However, it may not necessarily be true, given that there is at least the possibility that one modality provides all features that explain the topographic organization of the brain while all other aspects may agree or not but do not provide additional information on 
brain organization. Moreover, several important caveats need to be addressed in a systematic manner, before this proposition may be accepted as the fundamental truth about the topographic organization of the brain.

Most fundamental is the current lack of knowledge about the interrelation between features and the specific details of border co-localization. This in turn suggests, that there is no way to determine, to which degree different features may be considered redundant, convergent, or complementary in the context of human brain mapping and ultimately which information is needed to progress towards a multi-modal definition of cortical areas that also transfers well to other, non-investigated features.

To illustrate this point, we could suppose several neurobiological features that are measured by different techniques, in different subjects, using different models. Yet, they may reflect fundamentally only one aspect of the brain's spatial heterogeneity. Consequently, the parcellations obtained from these modalities will only differ from each other due to method-inherent artifacts or biases, as well as noise in the data. In this context, we note that that even in this scenario, multimodal (or multi-feature) parcellation has one massive advantage, namely the fact that convergence across features will increase faith in the obtained results in the presence of noisy data. That is, modules (or their borders) that converge across different features should always be considered more robust to random and systematic artifacts than those based on only a single one. Combining three features that reflect the same underlying differentiation certainly yields "better" maps in the technical sense. Nevertheless, the transferability of the obtained modules to other modalities may not be superior to that of maps derived from any single feature, given that the features represent redundant rather than complementary information. Consequently, mapping the brain based on a set of features that are reflecting different aspects of brain organization, ideally with multiple features reflecting any aspect, should be preferable if indeed it can be shown that each of these aspects of brain organization reveal a meaningful regional differentiation. As noted, however, there is currently no way to realize this goal in a principled, empirically grounded manner, given that our knowledge on the relationship between features is extremely limited. This also explains the appeal of resorting to structure, function and connectivity as the three aspects of interest given their (apparent) distinctiveness.

The question of which features should be combined to best reflect the topographic organization of the brain, and in fact, whether there is something like a best reflection thus needs further investigation. Systematically comparing and combining maps provided by features such as restingstate functional connectivity, fiber connections proxied by diffusion tractography, structural covariance, meta-analytic coactivation mapping, activation by a wide range of tasks and relative myelination should definitely provide a valuable step towards understanding cortical heterogeneity. Up to now, however, studies mapping the brain using multiple features are rare (some notable exceptions are provided by Glasser et al., 2016; Genon et al., 2016; Kelly et al., 2012; Wang et al., 2015) and none have combined all of these aspects.

How multi-modal parcellation based on complementary features should ideally be pursued is another open question. Fundamentally, two different approaches may be distinguished and both should provide interesting opportunities for multi-modal parcellation. The different features for each voxel could be combined into a vector and then parcellation, by border detection or clustering, could be performed on this multi-modal feature vector. Alternatively, cortical modules and their borders first may be defined in each modality, i.e., on each feature, individually and then integrated into a common map, e.g., by superimposition of borders or computing the intersecting volumes. Both approaches come with practical and conceptual challenges. For example, many features are 
themselves high-dimensional (such as the whole-brain coactivation profiles), while others, like relative myelination are scalar. Scaling, variance and signal to noise ratio should also be vastly different across features. Consequently, combining all features into a well-behaved feature vector that gives comparable weight to any individual aspect of brain organization remains a major challenge. Similarly, parcellations obtained from each individual feature will, without doubt, be more noisy than a consensus analysis, and hence integration of multiple parcellations is likewise non-trivial. Given differential susceptibility to specific bias and artifacts, it may be expected that borders revealed by different features are rarely in complete congruence. Likewise, it can be expected that particularly at the more fine-grained level, some borders are revealed by one feature but not the other.

The question thus arises, whether to focus on the convergence between parcellations, potentially throwing away relevant information, to weigh some modalities higher than others in certain areas based on prior knowledge, resulting in a semi-automated and hence potentially biased approach, or to consider each intersection between the different maps as a separate module. The latter has some theoretical appeal, as it would follow the idea that a relevant change in one feature should be sufficient to form a new module, i.e., that the topographic organization of the brain is formed by the intersections between multiple semi-overlapping maps. However, this approach may yield an extremely high number of spurious modules formed by spatial discrepancies among delineated borders that might simply be due to noise.

\section{Outgoing considerations}

Here we reviewed the idea of topographic organization in the (human) brain and particularly the cerebral cortex from the historical perspective before discussing the appeal and drawback of the current conceptualization of cortical areas. We then proposed a revised concept that is based on the idea of location-specific multidimensional feature vectors and outline, how cortical areas may be understood in such framework. Finally, we related current approaches for uni- and multi-modal (in-vivo) brain parcellation to this conceptual backdrop. We would like to close by raising two additional questions that are not directly in the scope of this overview but will become relevant in the future of human brain mapping.

One of the major questions regarding the human brain pertains to the number of cortical areas it is made up of. While estimates such as "200-300 areas" are commonplace, we would argue that this question is somewhat ill-posed in a hierarchically organized system. As we have argued above, the brain may be formed by a multi-hierarchical system in which cortical columns form the basic units and no two of them are completely alike in all features. Yet, most neighboring columns are similar enough so that they may be considered to form units. The borders between these units, in turn, are at least in some features pronounced enough to allow for a distinction. Still several of these units are similar to each other in most features and together form larger modules, which in turn are more separated from each other by borders that reflect more pronounced changes and are detectable in more features. Yet again, many of these modules may form larger areas and finally broader networks. Ultimately, there may be several useful resolutions depending on the particular question. A multilevel approach may also help to address the second question we discuss next which is that of inter-individual variability.

It is widely established that there is inter-individual variability in the size and exact location of cortical areas, as well as their connectivity with other areas and functional response. Yet, in spite of 
the marked variability of basically every aspect of cortical organization, one of the almost dogmatic assumptions of human brain mapping is that all people have the same areas. In other words, while almost everybody would agree that cortical areas can vary across individuals in all possible aspects, there seems to be a consensus that these should still be considered the same areas. We regard this predicament, in particular with respect to the poorly understood and highly variable organization of brain regions such as the prefrontal cortex, as one of the most important challenges in systems neuroscience, as it may be argued that human brain organization cannot be understood without understanding human brain variability.

Acknowledgements: SBE is supported by the Deutsche Forschungsgemeinschaft (DFG, EI 816/4-1, El 816/6-1), the National Institute of Mental Health (R01-MH074457), the Helmholtz Portfolio Theme "Supercomputing and Modeling for the Human Brain" and and the European Union's Horizon 2020 Research and Innovation Programme under Grant Agreement No. 7202070 (HBP SGA1). BTTY is supported by Singapore MOE Tier 2 (MOE2014-T2-2-016), NUS Strategic Research (DPRT/944/09/14), NUS SOM Aspiration Fund (R185000271720), Singapore NMRC (CBRG14nov007, NMRC/CG/013/2013) and NUS YIA. 


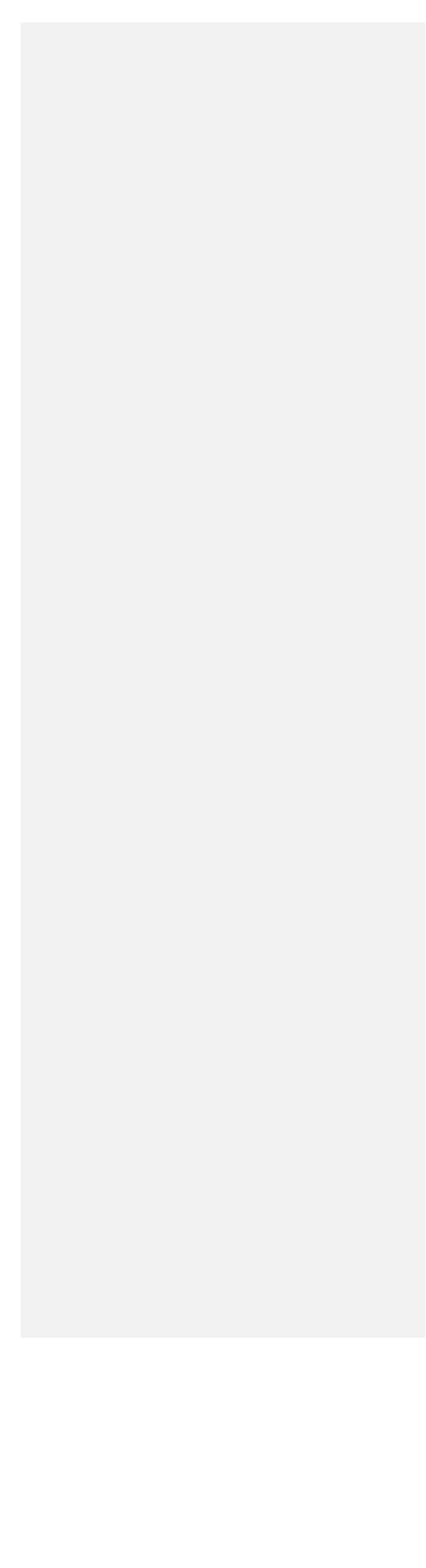




\section{References}

Alvarez JA, Emory E. (2006) Executive function and the frontal lobes: a meta-analytic review. Neuropsychol Rev. 16(1):17-42. Review.

Amunts K, Zilles K (2015) Architectonic Mapping of the Human Brain beyond Brodmann Neuron 88(6): 1086-1107

Amunts K, Hawrylycz MJ, Van Essen DC, Van Horn JD, Harel N, Poline JB, De Martino F, Bjaalie JG, Dehaene-Lambertz G, Dehaene S, Valdes-Sosa P, Thirion B, Zilles K, Hill SL, Abrams MB, Tass PA, Vanduffel W, Evans AC, Eickhoff SB. (2014) Interoperable atlases of the human brain. Neuroimage. 99:525-32.

Amunts K, Lenzen M, Friederici AD, Schleicher A, Morosan P, Palomero-Gallagher N, Zilles K. (2010) Broca's region: novel organizational principles and multiple receptor mapping. PLoS Biol. 8(9). pii: e1000489.

Bailey P, von Bonin G (1951) The Isocortex of Man, Univ. of Illinois Press, Urbana

Behrens TE, Johansen-Berg H, Woolrich MW, Smith SM, Wheeler-Kingshott CA, Boulby PA, Barker GJ, Sillery EL, Sheehan K, Ciccarelli O, Thompson AJ, Brady JM, Matthews PM. (2003) Non-invasive mapping of connections between human thalamus and cortex using diffusion imaging. Nat Neurosci. 6(7):750-7.

Bellec P, Benhajali Y, Carbonell F, Dansereau C, Albouy G, Pelland M, Craddock C, Collignon O, Doyon J, Stip E, Orban P. (2015) Impact of the resolution of brain parcels on connectome-wide association studies in fMRI. Neuroimage. 123:212-28.

Bellec P, Rosa-Neto P, Lyttelton OC, Benali H, Evans AC. (2010) Multi-level bootstrap analysis of stable clusters in resting-state fMRI. Neuroimage. 51(3):1126-39.

Biswal BB, Mennes M, Zuo XN, Gohel S, Kelly C, Smith SM, Beckmann CF, Adelstein JS, Buckner RL, Colcombe S, Dogonowski AM, Ernst M, Fair D, Hampson M, Hoptman MJ, Hyde JS, Kiviniemi VJ, Kötter R, Li SJ, Lin CP, Lowe MJ, Mackay C, Madden DJ, Madsen KH, Margulies DS, Mayberg HS, McMahon K, Monk CS, Mostofsky SH, Nagel BJ, Pekar JJ, Peltier SJ, Petersen SE, Riedl V, Rombouts SA, Rypma B, Schlaggar BL, Schmidt S, Seidler RD, Siegle GJ, Sorg C, Teng GJ, Veijola J, Villringer A, Walter M, Wang L, Weng XC, Whitfield-Gabrieli S, Williamson $\mathrm{P}$, Windischberger C, Zang YF, Zhang HY, Castellanos FX, Milham MP. (2010) Toward discovery science of human brain function. PNAS. 107(10):4734-9.

Bludau S, Eickhoff SB, Mohlberg H, Caspers S, Laird AR, Fox PT, Schleicher A, Zilles K, Amunts K. Cytoarchitecture, probability maps and functions of the human frontal pole. Neuroimage. $93 \mathrm{Pt}$ 2:260-75. doi: 10.1016/j.neuroimage.2013.05.052.

Bludau S, Bzdok D, Gruber O, Kohn N, Riedl V, Sorg C, Palomero-Gallagher N, Müller VI, Hoffstaedter F, Amunts K, Eickhoff SB. (2016) Medial Prefrontal Aberrations in Major Depressive Disorder Revealed by Cytoarchitectonically Informed Voxel-Based Morphometry. Am J Psychiatry. 173(3):291-8.

Bullmore E, Sporns O. (2012) The economy of brain network organization. Nat Rev Neurosci. 13(5):336-49. doi: 10.1038/nrn3214. Review.

Burton H, Fabri M, Alloway K. (1995) Cortical areas within the lateral sulcus connected to cutaneous representations in areas $3 \mathrm{~b}$ and 1 : a revised interpretation of the second somatosensory area in macaque monkeys. J Comp Neurol. 355(4):539-62. 
Bzdok D, Langner R, Schilbach L, Jakobs O, Roski C, Caspers S, Laird AR, Fox PT, Zilles K, Eickhoff SB. (2013) Characterization of the temporo-parietal junction by combining data-driven parcellation, complementary connectivity analyses, and functional decoding. Neuroimage. 81:38192. doi: 10.1016/j.neuroimage.2013.05.046.

Bzdok D, Heeger A, Langner R, Laird AR, Fox PT, Palomero-Gallagher N, Vogt BA, Zilles K, Eickhoff SB. (2015) Subspecialization in the human posterior medial cortex. Neuroimage. 106:5571.

Bzdok D, Laird AR, Zilles K, Fox PT, Eickhoff SB. (2013) An investigation of the structural, connectional, and functional subspecialization in the human amygdala. Hum Brain Mapp. 34(12):3247-66.

Caspers S, Eickhoff SB, Rick T, von Kapri A, Kuhlen T, Huang R, Shah NJ, Zilles K. (2011) Probabilistic fibre tract analysis of cytoarchitectonically defined human inferior parietal lobule areas reveals similarities to macaques. Neuroimage. 58(2):362-80.

Caspers S, Eickhoff SB, Zilles K, Amunts K. (2013) Microstructural grey matter parcellation and its relevance for connectome analyses. Neuroimage. 80:18-26.

Carmichael ST, Price JL. (1994) Architectonic subdivision of the orbital and medial prefrontal cortex in the macaque monkey. J Comp Neurol. 346(3):366-402.

Cerliani L, D'Arceuil H, Thiebaut de Schotten M. (2016) Connectivity-based parcellation of the macaque frontal cortex, and its relation with the cytoarchitectonic distribution described in current atlases. Brain Struct Funct. 2016 Epub ahead of print

Cieslik EC, Zilles K, Caspers S, Roski C, Kellermann TS, Jakobs O, Langner R, Laird AR, Fox PT, Eickhoff SB. (2013) Is there "one" DLPFC in cognitive action control? Evidence for heterogeneity from co-activation-based parcellation. Cereb Cortex. 23(11):2677-89.

Clos M, Amunts K, Laird AR, Fox PT, Eickhoff SB. (2013) Tackling the multifunctional nature of Broca's region meta-analytically: co-activation-based parcellation of area 44. Neuroimage. 83:17488

Craddock RC, Jbabdi S, Yan CG, Vogelstein JT, Castellanos FX, Di Martino A, Kelly C, Heberlein K, Colcombe S, Milham MP. (2013) Imaging human connectomes at the macroscale. Nat Methods. 10(6):524-39.

Deco G, Tononi G, Boly M, Kringelbach ML. (2015) Rethinking segregation and integration: contributions of whole-brain modelling. Nat Rev Neurosci. 16(7):430-9

Desikan RS, Ségonne F, Fischl B, Quinn BT, Dickerson BC, Blacker D, Buckner RL, Dale AM, Maguire RP, Hyman BT, Albert MS, Killiany RJ. (2006) An automated labeling system for subdividing the human cerebral cortex on MRI scans into gyral based regions of interest. Neuroimage. 31(3):968-80.

Disbrow E, Litinas E, Recanzone GH, Padberg J, Krubitzer L. (2003) Cortical connections of the second somatosensory area and the parietal ventral area in macaque monkeys. J Comp Neurol. 4;462(4):382-99.

Donahue, C.J., Sotiropoulos, S.N., Jbabdi, S., Hernandez-Fernandez, M., Behrens, T.E., Dyrby, T.B., Coalson, T., Kennedy, H., Knoblauch, K., Van Essen, D.C., Glasser, M.F., 2016. Using Diffusion Tractography to Predict Cortical Connection Strength and Distance: A Quantitative Comparison with Tracers in the Monkey. The Journal of Neuroscience 36 (25), 6758-6770 
Eickhoff SB, Stephan KE, Mohlberg H, Grefkes C, Fink GR, Amunts K, Zilles K. (2005) A new SPM toolbox for combining probabilistic cytoarchitectonic maps and functional imaging data.

Neuroimage. 25(4):1325-35.

Eickhoff S, Walters NB, Schleicher A, Kril J, Egan GF, Zilles K, Watson JD, Amunts K. (2005) High-resolution MRI reflects myeloarchitecture and cytoarchitecture of human cerebral cortex. Hum Brain Mapp. 24(3):206-15.

Eickhoff SB, Grefkes C. (2011) Approaches for the integrated analysis of structure, function and connectivity of the human brain. Clin EEG Neurosci. 42(2):107-21. Review.

Eickhoff SB, Rottschy C, Kujovic M, Palomero-Gallagher N, Zilles K. (2008) Organizational principles of human visual cortex revealed by receptor mapping. Cereb Cortex. 18(11):2637-45. doi: 10.1093/cercor/bhn024.

Eickhoff SB, Thirion B, Varoquaux G, Bzdok D. (2015) Connectivity-based parcellation: Critique and implications. Hum Brain Mapp. 36(12):4771-92.

Eickhoff SB, Grefkes C, Fink GR, Zilles K. (2008). Functional lateralization of face, hand, and trunk representation in anatomically defined human somatosensory areas. Cereb Cortex. 18(12):282030. doi: 10.1093/cercor/bhn039.

Eickhoff SB, Etkin A. (2016) Going Beyond Finding the "Lesion": A Path for Maturation of Neuroimaging. Am J Psychiatry. 173(3):302-3.

Evans AC. (2013) Networks of anatomical covariance. Neuroimage. 80:489-504.

Felleman DJ, Van Essen DC (1991) Distributed hierarchical processing in the primate cerebral cortex. Cereb Cortex. 1(1):1-47.

Fan L, Li H, Zhuo J, Zhang Y, Wang J, Chen L, Yang Z, Chu C, Xie S, Laird AR, Fox PT, Eickhoff SB, Yu C, Jiang T. (2016) The Human Brainnetome Atlas: A New Brain Atlas Based on Connectional Architecture. Cereb Cortex. 26(8):3508-26.

Finger S (2004). Minds Behind the Brain: A History of the Pioneers and Their Discoveries. New York: Oxford University Press. p. 29. ISBN 0195181824.

Finn ES, Shen X, Scheinost D, Rosenberg MD, Huang J, Chun MM, Papademetris X, Constable RT (2015) Functional connectome fingerprinting: identifying individuals using patterns of brain connectivity. Nat Neurosci. 18(11):1664-71

Finn ES, Constable RT, Individual variation in functional brain connectivity: implications for personalized approaches to psychiatric disease. (2016) Dialogues Clin Neurosci. 18(3):277-287.

Fox MD, Snyder AZ, Vincent JL, Corbetta M, Van Essen DC, Raichle ME. (2005) The human brain is intrinsically organized into dynamic, anticorrelated functional networks. PNAS. 102(27):9673-8.

Fox PT, Friston KJ. (2012) Distributed processing; distributed functions? Neuroimage. 61(2):40726. Doi

Fox PT, Lancaster JL, Laird AR, Eickhoff SB. (2014) Meta-analysis in human neuroimaging: computational modeling of large-scale databases. Annu Rev Neurosci. 37:409-34.

Foxley S, Jbabdi S, Clare S, Lam W, Ansorge O, Douaud G, Miller K. (2014) Improving diffusionweighted imaging of post-mortem human brains: SSFP at 7 T. Neuroimage. 102 Pt 2:579-89

Genon S, Li H, Fan L, Müller VI, Cieslik EC, Hoffstaedter F, Reid AT, Langner R, Grefkes C, Fox PT, Moebus S, Caspers S, Amunts K, Jiang T, Eickhoff SB. (2016) The Right Dorsal Premotor Mosaic: Organization, Functions, and Connectivity. Cereb Cortex. Epub ahead of print

Glasser MF, Coalson TS, Robinson EC, Hacker CD, Harwell J, Yacoub E, Ugurbil K, Andersson J, Beckmann CF, Jenkinson M, Smith SM, Van Essen DC. (2016) A multi-modal parcellation of human cerebral cortex. Nature. 536(7615):171-8. 
Gordon EM, Laumann TO, Adeyemo B, Huckins JF, Kelley WM, Petersen SE. (2016) Generation and Evaluation of a Cortical Area Parcellation from Resting-State Correlations. Cereb Cortex. 26(1):288-303.

Grinvald, A. , Slovin, H., Vanzetta, I. (2000), Non-invasive visualization of cortical columns by fMRI, Nature Neuroscience 3(2):105-108

Henssen A, Zilles K, Palomero-Gallagher N, Schleicher A, Mohlberg H, Gerboga F, Eickhoff SB, Bludau S, Amunts K. (2016) Cytoarchitecture and probability maps of the human medial orbitofrontal cortex. Cortex. 75:87-112.

Horton JC, Adams DL. (2005) The cortical column: a structure without a function. Philos Trans R Soc Lond B Biol Sci. 360(1456):837-62.

Hubel DH, Wiesel TN. (1968) Receptive fields and functional architecture of monkey striate cortex. J Physiol. 195(1):215-43.

Johansen-Berg H, Behrens TE, Robson MD, Drobnjak I, Rushworth MF, Brady JM, Smith SM, Higham DJ, Matthews PM. (2004) Changes in connectivity profiles define functionally distinct regions in human medial frontal cortex. PNAS. 101(36):13335-40.

Kaiser M. (2011) A tutorial in connectome analysis: topological and spatial features of brain networks. Neuroimage. 57(3):892-907

Kelly C, Toro R, Di Martino A, Cox CL, Bellec P, Castellanos FX, Milham MP. (2012) A convergent functional architecture of the insula emerges across imaging modalities. Neuroimage. 61(4):112942.

Krubitzer L, Clarey J, Tweedale R, Elston G, Calford M. (1995) A redefinition of somatosensory areas in the lateral sulcus of macaque monkeys. J Neurosci. 15(5 Pt 2):3821-39.

Krubitzer L, Kaas J. (2005) The evolution of the neocortex in mammals: how is phenotypic diversity generated? Curr Opin Neurobiol. 15(4):444-53.

Langner R, Eickhoff SB. (2013) Sustaining attention to simple tasks: a meta-analytic review of the neural mechanisms of vigilant attention. Psychol Bull. 139(4):870-900.

Lancaster JL, Woldorff MG, Parsons LM, Liotti M, Freitas CS, Rainey L, Kochunov PV, Nickerson D, Mikiten SA, Fox PT. (2000) Automated Talairach atlas labels for functional brain mapping. Hum Brain Mapp. 10(3):120-31.

Large I, Bridge H, Ahmed B, Clare S, Kolasinski J, Lam WW, Miller KL, Dyrby TB, Parker AJ, Smith JE, Daubney G, Sallet J, Bell AH, Krug K. (2016) Individual Differences in the Alignment of Structural and Functional Markers of the V5/MT Complex in Primates. Cereb Cortex. 26(10):392844.

Leiner, H.C., Leiner, A.L., Dow, R.S., (1991) The human cerebro-cerebellar system: its computing, cognitive, and language skills, Behavioural Brain Research 44(2): 113-128.

Levy, R, Goldman-Rakic, P.S., (2000) Segregation of working memory functions within the dorsolateral prefrontal cortex Experimental Brain Research 133(1):23-32

Loewe K, Donohue SE, Schoenfeld MA, Kruse R and Borgelt C (2016). Memory-efficient analysis of dense functional connectomes. Front. Neuroinform. 10:50

Mah YH, Husain M, Rees G, Nachev P. (2014) Human brain lesion-deficit inference remapped. Brain. 137(Pt 9):2522-31.

Mangin JF, Lebenberg J, Lefranc S, Labra N, Auzias G, Labit M, Guevara M, Mohlberg H, Roca P, Guevara P, Dubois J, Leroy F, Dehaene-Lambertz G, Cachia A, Dickscheid T, Coulon O, Poupon $\mathrm{C}$, Rivière $\mathrm{D}$, Amunts $\mathrm{K}$, Sun $\mathrm{ZY}$. (2016) Spatial normalization of brain images and beyond. Med Image Anal. 33:127-33.

Maier-Hein K, Neher P, Houde JC, Cote MA, Garyfallidis E, Zhong J, Chamberland M, Yeh FC, Lin Y, Ji Q, Reddick WE, Glass JO, Chen DQ, Feng Y, Gao C, Wu Y, Ma J, Renjie H, Li Q, Westin CF, Deslauriers-Gauthier S, Gonzalez J, Paquette M, St-Jean S, Girard G, Rheault F, Sidhu J, Tax 
CM, Guo F, Mesri HY, David S, Froeling M, Heemskerk AM, Leemans A, Bore A, Pinsard B, Bedetti C, Desrosiers M, Brambati S, Doyon J, Sarica A, Vasta R, Cerasa A, Quattrone A, Yeatman J, Khan AR, Hodges W, Alexander S, Romascano D, Barakovic M, Auria A, Esteban O, Lemkaddem A, Thiran JP, Cetingul HE, Odry BL, Mailhe B, Nadar M, Pizzagalli F, Prasad G, Villalon-Reina J, Galvis J, Thompson P, Requejo F, Laguna P, Lacerda L, Barrett R, Dell'Acqua F, Catani M, Petit L, Caruyer E, Daducci A, Dyrby T, Holland-Letz T, Hilgetag C, Stieltjes B Descoteaux M. (2016) Tractography- based connectomes are dominated by false-positive connections. BioarXiv, http://dx.doi.org/10.1101/084137

N. T. Markov, M. M. Ercsey-Ravasz, A. R. Ribeiro Gomes, C. Lamy, L. Magrou, J. Vezoli, P. Misery, A. Falchier, R. Quilodran, M. A. Gariel, J. Sallet, R. Gamanut, C. Huissoud, S. Clavagnier, P. Giroud, D. Sappey-Marinier, P. Barone, C. Dehay, Z. Toroczkai, K. Knoblauch, D. C. Van Essen, H. Kennedy; (2012) A Weighted and Directed Interareal Connectivity Matrix for Macaque Cerebral Cortex. Cereb Cortex 24 (1): 17-36.

Mars RB, Sallet J, Schüffelgen U, Jbabdi S, Toni I, Rushworth MF. (2012) Connectivity-based subdivisions of the human right "temporoparietal junction area": evidence for different areas participating in different cortical networks. Cereb Cortex. 22(8):1894-903

McIntosh AR, Mišić B. (2013) Multivariate statistical analyses for neuroimaging data. Annu Rev Psychol. 64:499-525.

Miyake A, Friedman NP, Emerson MJ, Witzki AH, Howerter A, Wager TD. (2000) The unity and diversity of executive functions and their contributions to complex "Frontal Lobe" tasks: a latent variable analysis. Cogn Psychol. 41(1):49-100.

Muhle-Karbe PS, Derrfuss J, Lynn MT, Neubert FX, Fox PT, Brass M, Eickhoff SB. (2016) CoActivation-Based Parcellation of the Lateral Prefrontal Cortex Delineates the Inferior Frontal Junction Area. Cereb Cortex. 26(5):2225-41.

Mountcastle $V$ (1957) Modality and topographic properties of single neurons of cat's somatic sensory cortex. J. Neurophysiol. 20:408-434.

Moyer D, Gutman B, Prasad G, Faskowitz J, Ver Steeg G, Thompson PM. (2015) Blockmodels for connectome analysis. In 11th International Symposium on Medical Information Processing and Analysis (SIPAIM 2015), International Society for Optics and Photonics, 96810A-96810A.

Najafi M, McMenamin BW, Simon JZ, Pessoa L. (2016) Overlapping communities reveal rich structure in large-scale brain networks during rest and task conditions. Neurolmage. 135:92-106

Nooner KB, Colcombe SJ, Tobe RH, Mennes M, Benedict MM, Moreno AL, Panek LJ, Brown S, Zavitz ST, Li Q, Sikka S, Gutman D, Bangaru S, Schlachter RT, Kamiel SM, Anwar AR, Hinz CM, Kaplan MS, Rachlin AB, Adelsberg S, Cheung B, Khanuja R, Yan C, Craddock CC, Calhoun V, Courtney W, King M, Wood D, Cox CL, Kelly AM, Di Martino A, Petkova E, Reiss PT, Duan N, Thomsen D, Biswal B, Coffey B, Hoptman MJ, Javitt DC, Pomara N, Sidtis JJ, Koplewicz HS, Castellanos FX, Leventhal BL, Milham MP. (2012) The NKI-Rockland Sample: A Model for Accelerating the Pace of Discovery Science in Psychiatry. Front Neurosci. 6:152

Orban GA, Van Essen D, Vanduffel W. (2004) Comparative mapping of higher visual areas in monkeys and humans. Trends Cogn Sci. 8(7):315-24.

Pauli WM, O'Reilly RC, Yarkoni T, Wager TD. (2016) Regional specialization within the human striatum for diverse psychological functions. PNAS 113(7):1907-12.

Palomero-Gallagher N, Eickhoff SB, Hoffstaedter F, Schleicher A, Mohlberg H, Vogt BA, Amunts K, Zilles K. (2015) Functional organization of human subgenual cortical areas: Relationship between architectonical segregation and connectional heterogeneity. Neuroimage.;115:177-90.

Park HJ, Friston K. (2013) Structural and functional brain networks: from connections to cognition. Science. 342(6158):1238411. doi: 10.1126/science.1238411.

Poldrack RA, Kittur A, Kalar D, Miller E, Seppa C, Gil Y, Parker DS, Sabb FW, Bilder RM. (2011) The cognitive atlas: toward a knowledge foundation for cognitive neuroscience. Front Neuroinform. $6 ; 5: 17$ 
Raichle ME (2009) A brief history of human brain mapping. Trends Neurosci. 32(2):118-26.

Ray KL, Zald DH, Bludau S, Riedel MC, Bzdok D, Yanes J, Falcone KE, Amunts K, Fox PT, Eickhoff SB, Laird AR. (2015) Co-activation based parcellation of the human frontal pole. Neuroimage. 123:200-11

Reid AT, Lewis J, Bezgin G, Khundrakpam B, Eickhoff SB, McIntosh AR, Bellec P, Evans AC. (2016) A cross-modal, cross-species comparison of connectivity measures in the primate brain. Neuroimage. 125:311-31.

Reid AT, Hoffstaedter F, Gong G, Laird AR, Fox P, Evans AC, Amunts K, Eickhoff SB. (2017) A seed-based cross-modal comparison of brain connectivity measures. Brain Struct Funct. In press

Rockland KS, Pandya DN. (1979) Laminar origins and terminations of cortical connections of the occipital lobe in the rhesus monkey. Brain Res. 179(1):3-20.

Robinson EC, Jbabdi S, Glasser MF, Andersson J, Burgess GC, Harms MP, Smith SM, Van Essen DC, Jenkinson M. (2014) MSM: a new flexible framework for multi-modal Surface Matching. Neuroimage. 100:414-26.

Rorden C, Karnath $\mathrm{H}-\mathrm{O}$ (2004) Using human brain lesions to infer function: a relic from a past era in the fMRI age? Nature Reviews Neuroscience 5:812-819.

Rosenberg MD, Finn ES, Scheinost D, Papademetris X, Shen X, Constable RT, Chun MM. (2016a) A neuromarker of sustained attention from whole-brain functional connectivity. Nat Neurosci. 19(1):165-71.

Rosenberg MD, Zhang S, Hsu WT, Scheinost D, Finn ES, Shen X, Constable RT, Li CS, Chun MM., (2016) Methylphenidate Modulates Functional Network Connectivity to Enhance Attention. J Neurosci. 36(37):9547-57

Ross WD, Grossberg S, Mingolla E. (2000) Visual cortical mechanisms of perceptual grouping: interacting layers, networks, columns, and maps. Neural Netw. 13(6):571-88.

Rottschy C, Eickhoff SB, Schleicher A, Mohlberg H, Kujovic M, Zilles K, Amunts K. (2007) Ventral visual cortex in humans: cytoarchitectonic mapping of two extrastriate areas. Hum Brain Mapp. 28(10):1045-59.

Sallet J, Mars RB, Noonan MP, Neubert FX, Jbabdi S, O'Reilly JX, Filippini N, Thomas AG, Rushworth MF. (2013) The organization of dorsal frontal cortex in humans and macaques. J Neurosci. 33(30):12255-74.

Sampaio-Baptista C, Khrapitchev AA, Foxley S, Schlagheck T, Scholz J, Jbabdi S, DeLuca GC, Miller KL, Taylor A, Thomas N, Kleim J, Sibson NR, Bannerman D, Johansen-Berg H. (2013) Motor skill learning induces changes in white matter microstructure and myelination. J Neurosci. 33(50):19499-503.

Scannell JW, Burns GA, Hilgetag CC, O'Neil MA, Young MP. (1999) The connectional organization of the cortico-thalamic system of the cat. Cereb Cortex. 9(3):277-99.

Selemon, L.D., Goldman-Rakic, P.S.,(1985) Longitudinal topography and interdigitation of corticostriatal projections in the rhesus monkey, Journal of Neuroscience, 5(3):776-794

Shen X, Tokoglu F, Papademetris X, Constable RT. (2013) Groupwise whole-brain parcellation from resting-state fMRI data for network node identification. Neuroimage. 82:403-15.

Shehzad Z, Kelly C, Reiss PT, Cameron Craddock C, Emerson JW, McMahon K, Copland DA, Castellanos FX, Milham MP. (2014) A multivariate distance-based analytic framework for connectome-wide association studies. Neuroimage. 93 Pt 1:74-94. 
Sotiras A, Resnick SM, Davatzikos C. (2015) Finding imaging patterns of structural covariance via Non-Negative Matrix Factorization. Neuroimage. 108:1-16.

Sporns O. (2011) The human connectome: a complex network. Ann N Y Acad Sci. 1224:109-25

Sporns O, Chialvo DR, Kaiser M, Hilgetag CC. (2004) Organization, development and function of complex brain networks. Trends Cogn Sci. 8(9):418-25.

Stikov N, Campbell JS, Stroh T, Lavelée M, Frey S, Novek J, Nuara S, Ho MK, Bedell BJ, Dougherty RF, Leppert IR, Boudreau M, Narayanan S, Duval T, Cohen-Adad J, Picard PA, Gasecka A, Côté D, Pike GB. (2015) In vivo histology of the myelin g-ratio with magnetic resonance imaging. Neuroimage. 118:397-405.

Talairach J, Tournoux P (1988) Co-planar stereotaxic atlas of the human brain. 3-Dimensional proportional system: an approach to cerebral imaging. Thieme, Stuttgart

Thirion B, Varoquaux G, Dohmatob E, Poline JB. (2014) Which fMRI clustering gives good brain parcellations? Front Neurosci. 8:167.

Thiebaut de Schotten M, Urbanski M, Batrancourt B, Levy R, Dubois B, Cerliani L, Volle E. (2016) Rostro-caudal Architecture of the Frontal Lobes in Humans. Cereb Cortex. Epub ahead of print

Turner JA, Laird AR. (2012) The cognitive paradigm ontology: design and application. Neuroinformatics. 10(1):57-66.

Uğurbil K, Xu J, Auerbach EJ, Moeller S, Vu AT, Duarte-Carvajalino JM, Lenglet C, Wu X, Schmitter S, Van de Moortele PF, Strupp J, Sapiro G, De Martino F, Wang D, Harel N, Garwood M, Chen L, Feinberg DA, Smith SM, Miller KL, Sotiropoulos SN, Jbabdi S, Andersson JL, Behrens TE, Glasser MF, Van Essen DC, Yacoub E; WU-Minn HCP Consortium. (2013) Pushing spatial and temporal resolution for functional and diffusion MRI in the Human Connectome Project. Neuroimage. 80:80-104. doi: 10.1016/j.neuroimage.2013.05.012. Epub 2013 May 21

Ugurbil, K., (2016) What is feasible with imaging human brain function and connectivity using functional magnetic resonance imaging, Philosophical Transactions of the Royal Society B: Biological Sciences, 371(1705): Article number 20150361

Van Essen DC, Maunsell JH, Bixby JL. (1981) The middle temporal visual area in the macaque: myeloarchitecture, connections, functional properties and topographic organization. J Comp Neurol. 199(3):293-326.

Van Essen DC, Smith SM, Barch DM, Behrens TE, Yacoub E, Ugurbil K; WU-Minn HCP Consortium. (2013) The WU-Minn Human Connectome Project: an overview. Neuroimage. 80:6279.

Wang J, Yang Y, Fan L, Xu J, Li C, Liu Y, Fox PT, Eickhoff SB, Yu C, Jiang T. (2015) Convergent functional architecture of the superior parietal lobule unraveled with multi-modal neuroimaging approaches. Hum Brain Mapp. 36(1):238-57.

Zhang W, Wang J, Fan L, Zhang Y, Fox PT, Eickhoff SB, Yu C, Jiang T. (2016) Functional organization of the fusiform gyrus revealed with connectivity profiles. Hum Brain Mapp. 37(8):300316.

Wilms M, Eickhoff SB, Specht K, Amunts K, Shah NJ, Malikovic A, Fink GR. (2005) Human V5/MT+: comparison of functional and cytoarchitectonic data. Anat Embryol (Berl). 210(5-6):48595.

Wise SP, Boussaoud D, Johnson PB, Caminiti R. (1997) Premotor and parietal cortex: corticocortical connectivity and combinatorial computations. Annu Rev Neurosci. 20:25-42. 
Wu SS, Chang TT, Majid A, Caspers S, Eickhoff SB, Menon V. (2009) Functional heterogeneity of inferior parietal cortex during mathematical cognition assessed with cytoarchitectonic probability maps. Cereb Cortex. 19(12):2930-45.

Yacoub E, Harel N, Ugurbil K. (2008) High-field fMRI unveils orientation columns in humans. PNAS. 105(30):10607-12.

Yeo BT, Eickhoff SB. (2016) Systems neuroscience: A modern map of the human cerebral cortex. Nature. 536(7615):152-4.

Yeo BTI, Krienen FM, Sepulcre J, Sabuncu MR, Lashkari D, Hollinshead M, Roffman JL, Smoller JW, Zöllei L, Polimeni JR, Fischl B, Liu H, Buckner RL. (2011) The organization of the human cerebral cortex estimated by intrinsic functional connectivity. J Neurophysiol. 106(3):1125-65. doi: 10.1152/jn.00338.2011.

Yeo BTT, Krienen FM, Chee MWL, Buckner RL. (2014) Estimates of segregation and overlap of functional connectivity networks in the human cerebral cortex. Neuroimage, 88:212-227, 2014.

Yeo BTT, Krienen FM, Eickhoff SB, Yaakub SN, Fox PT, Buckner RL, Asplund CL, Chee MWL. (2015) Functional specialization and flexibility in human association cortex. Cerebral Cortex, 25:3654-3672,Yoshioka T, Blasdel GG, Levitt JB, Lund JS. (1996) Relation between patterns of intrinsic lateral connectivity, ocular dominance, and cytochrome oxidase-reactive regions in macaque monkey striate cortex. Cereb Cortex. 6(2):297-310.

Zalesky A, Fornito A, Harding IH, Cocchi L, Yücel M, Pantelis C, Bullmore ET. (2010) Whole-brain anatomical networks: does the choice of nodes matter? Neuroimage. 50(3):970-83.

Zeki S, McKeefry DJ, Bartels A, Frackowiak RS. (1998) Has a new color area been discovered? Nat Neurosci. 1(5):335-6.

Zielinski BA, Gennatas ED, Zhou J, Seeley WW. (2010) Network-level structural covariance in the developing brain. PNAS. 107(42):18191-6.

Zilles K, Palomero-Gallagher N. (2001) Cyto-, myelo-, and receptor architectonics of the human parietal cortex. Neuroimage. 14(1 Pt 2):S8-20.

Zilles K, Bacha-Trams M, Palomero-Gallagher N, Amunts K, Friederici AD. (2015) Common molecular basis of the sentence comprehension network revealed by neurotransmitter receptor fingerprints. Cortex. 63:79-89.

Zhou J, Gennatas ED, Kramer JH, Miller BL, Seeley WW. (2012) Predicting regional neurodegeneration from the healthy brain functional connectome. Neuron. 73(6):1216-27 